\title{
Sonourethrography in Evaluation of Abnormalities of Anterior Male Urethra.
}

\author{
Dr. Anand Hatgaonkar ${ }^{1}$, Dr. Pratibha Pendharkar ${ }^{2}$ \\ ${ }^{1}$ (Assistant Professor Department of Radiodiagnosis, Government Medical College Nagpur, Maharashtra, \\ India); ${ }^{2}$ (Ex-Dean, Professor and Head Department of Radiodiagnosis, Indira Gandhi Government Medical \\ College Nagpur, Maharashtra, India)
}

\begin{abstract}
Urethral pathologies are common problem affecting male anterior urethra. The male urethral imaging and pathology is not widespread in radiology literature because this part of the urinary tract is easily studied by urologists.

Retrograde urethrography $(R G U)$ and voiding cystourethrography have been the standard imaging studies for the evaluation of male anterior urethra, which involves the use of radiation and contrast medium to visualize luminal anatomy and gives limited information about periurethral structures. Radiation is harmful especially to the gonads which are frequently exposed during these examinations. Patients with urethral problems frequently and repeatedly require these examinations.

Sonourethrography with high-resolution ultrasound has an important role to play in the study of the diseases of the male urethra since it can detect pathology not visible on urethroscopy, specifically periurethral pathologies. As a dynamic, three-dimensional study, which can be repeated without radiation exposure, sonourethrography offers important advantages over conventional techniques. This study was undertaken to explore the uses of high-resolution ultrasound in evaluating abnormalities of the male anterior urethra.

Keywords: Anterior, Male, Sonourethrography, Stricture, Urethra.
\end{abstract}

\section{Introduction}

Urethral pathologies especially strictures are a common malady that affect male anterior urethra. The male urethral imaging and pathology is not widespread in the radiology literature because this part of the urinary tract is easily studied by urologists with clinical or endoscopic examinations. Imaging has an important role to play in the study of the diseases of the male urethra since it can detect pathology not visible on urethroscopy. The new imaging techniques in this area, such as sonography and MRI (Magnetic Resonance Imaging), can provide adjunct information that cannot be obtained with other modalities. [1]

Retrograde urethrography (RGU) and voiding cystourethrography have been the standard imaging studies for the male anterior urethra. Diagnostic imaging of the male urethra depends on these techniques, which involve the use of radiation and contrast medium to visualize luminal anatomy but gives limited information about periurethral structures.

In 1988 McAninch et al. [2] reported a new technique for imaging the male anterior urethra with highresolution ultrasound (Sonourethrography). The initial technique involved the use of a $5 \mathrm{MHz}$ linear array transducer applied to the dorsal surface of the penis. Images were obtained during retrograde instillation of normal saline.

As a dynamic, three-dimensional study, which can be repeated without radiation exposure, sonourethrography offers important advantages over conventional techniques. This study was undertaken to explore the uses of high-resolution ultrasound in evaluating abnormalities of the male anterior urethra.

\section{Material And Methods}

This study was carried out in the Department of Radiology, Indira Gandhi Government Medical College and Mayo Hospital, Nagpur over a period of 2 years from Jun 2003 to Jun 2005.

Sixty male patients with urinary complaints suggestive of urethral pathology were selected from the outpatient department. Patients with symptoms suggestive of acute urethritis were not included. In patients with recent instrumentation procedure was postponed for a week.

Sonourethrography (SUG) was performed using I) ESAOTE ultrasound \& color Doppler equipment and II) GE LOGIC 3 PRO ultrasound \& color Doppler equipment with a 5, 7.5 \& $10 \mathrm{mHz}$ transducers. With the patient in supine position, the glans was disinfected and a $12 \mathrm{~F}$ Foley catheter introduced such that the bulb laid in the fossa navicularis. The bulb was distended gently using $2 \mathrm{ml}$ normal saline. In the presence of meatal stenosis, the study was carried out while injecting saline directly with a syringe into the urethra. The penis was then cranially extended over the lower abdomen and ultrasonic gel was applied liberally to the ventral surface of the penis. Then 20 to $100 \mathrm{ml}$ of sterile normal saline was injected after taking care to exclude air bubbles. The 
penile urethra was visualized up to the penoscrotal junction with multiple longitudinal and transverse scans by placing the transducer on the ventral penile surface. Subsequently, the transducer was repositioned to visualize the proximal penile and distal bulbar urethra trans-scrotally and trans-perineally. The stricture length and diameter were determined using electronic caliper measurements. The duration of the procedure varied from 10$20 \mathrm{~min}$. Panoramic reconstruction of the sonographic images was done for better understanding of urethral pathologies.

\section{Observations And Result}

A total of 60 patients clinically presenting with urethral problems were selected for the study.

Average age of the patients in this study was 36.33 year. Maximum number of patients belonged to age group 31-40 years (Fig 1).

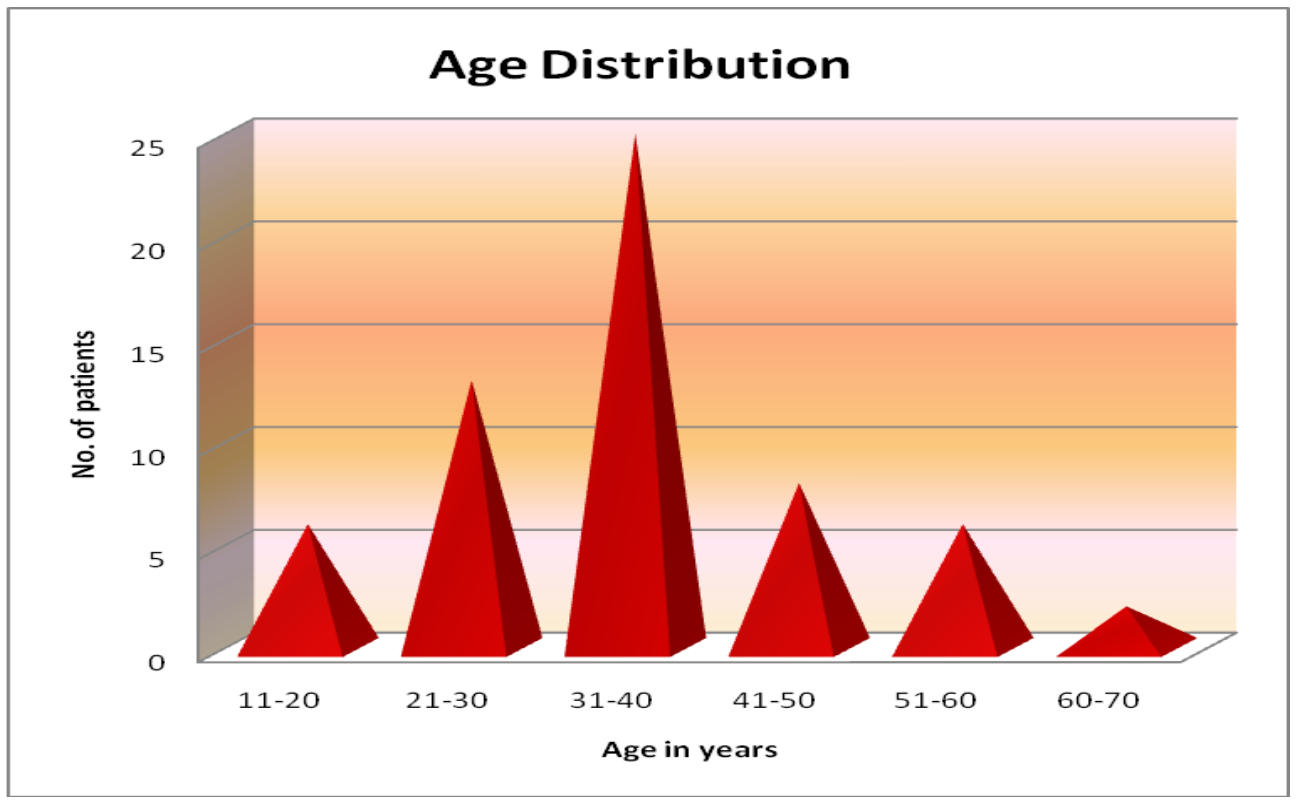

Fig-1: Age distribution of the patients for sonourethrography.

The SUG ruled out urethral lesion in 11 patients, and those were reported normal (Fig-2). Remaining 49 patients had definite signs of urethral abnormalities of varying type (Fig-4). In these 49 patients, 106 findings were demonstrated on sonourethrography (Table-1). Number of total findings far exceeded the number of patients as few patients had multiple findings.

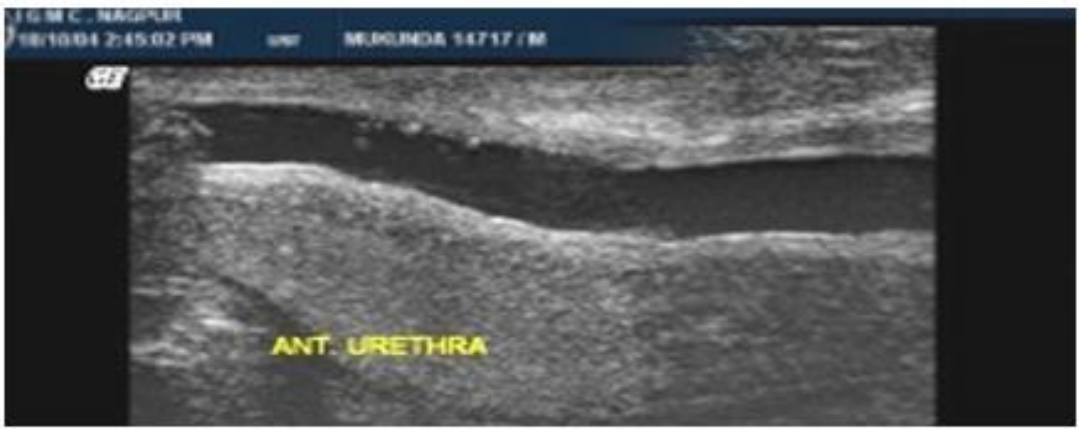

Fig-2: Normal sonourethrography appearance of anterior urethra longitudinal view.

Most common diagnosis was stricture. Total 51 strictures were demonstrated in 44 patients. Out of 44 patients single stricture was found in 31 patients and multiple or complex strictures in 13 patients. Out of 44 patients, 28 patients had short segment stricture, 3 patients had long segment stricture and 13 patients had complex/multiple strictures. Short segment strictures were in total 28 patients out of which 22 patients had bulbar short segment stricture. And six penile short segment strictures. In long segment strictures total three patients were identified, two with long segment penile stricture and one with long segment bulbar stricture found on SUG (Table-2). 
Sonourethrography in Evaluation of Abnormalities of Anterior Male Urethra.

Table-1 Distribution of patients according to Sonourethrography diagnosis

\begin{tabular}{|l|c|}
\hline \multicolumn{1}{|c|}{ Sonourethrography findings } & Total number \\
\hline Strictures & 51 \\
\hline False passage & 16 \\
\hline Diverticula + Syringocele & 04 \\
\hline Urethrocutaneous fistula & 01 \\
\hline Urethral mass & 01 \\
\hline Disruption of urethra & 02 \\
\hline Periurethral absseses & 04 \\
\hline Urethral calculus & 01 \\
\hline Periurethral haematoma/collection & 02 \\
\hline Intraluminal clot & 01 \\
\hline Urethritis & 23 \\
\hline Total findings & 106 \\
\hline
\end{tabular}

Out of 44 patients diagnosed with urethral stricture disease, sonography revealed periurethral fibrosis (PUF) in $34(56.70 \%)$ patients. Mild PUF was seen in seven patients (11.70\%), moderate in 13 patients $(21.70 \%)$ and extensive in 14 patients $(23.30 \%)$.

The next common diagnosis was urethritis, seen in 23 patients. Periurethral false passages were seen in 16 patients with one patient of urethrocutaneus fistula.

Urethral diverticula were seen in four patients including one patient having syringocele. Urethral mass was seen in one patient. One patient had intraluminal urethral calculus.

Five patients had trauma with fracture pelvis. We were unable to make out fractured bones on sonourethrography however urethral injury was well delineated with sonourethrography along with associated periurethral findings. Among these were two patients with disruption of bulbar urethra who had associated periurethral hematoma and collection (Fig-3). Total four patients had periurethral abscesses.
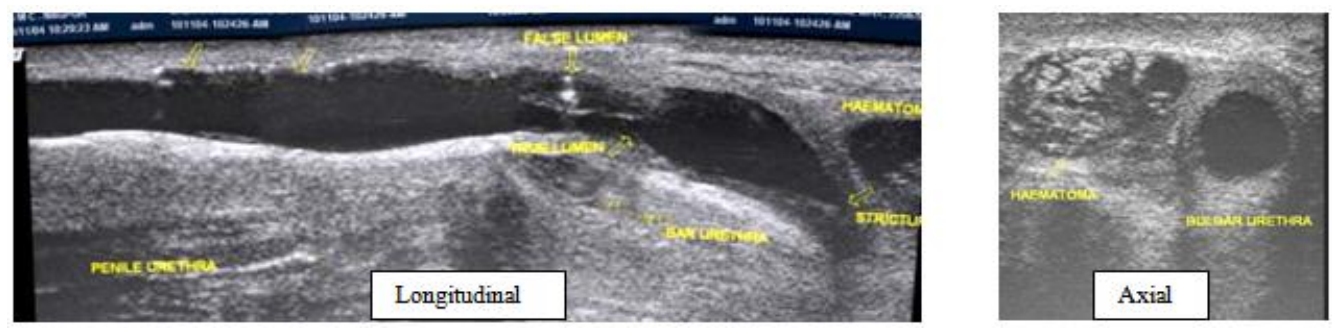

Fig-3: Post traumatic disruption at bulbo-membranous junction with periurethral hematoma and false lumen as seen on sonourethrography.

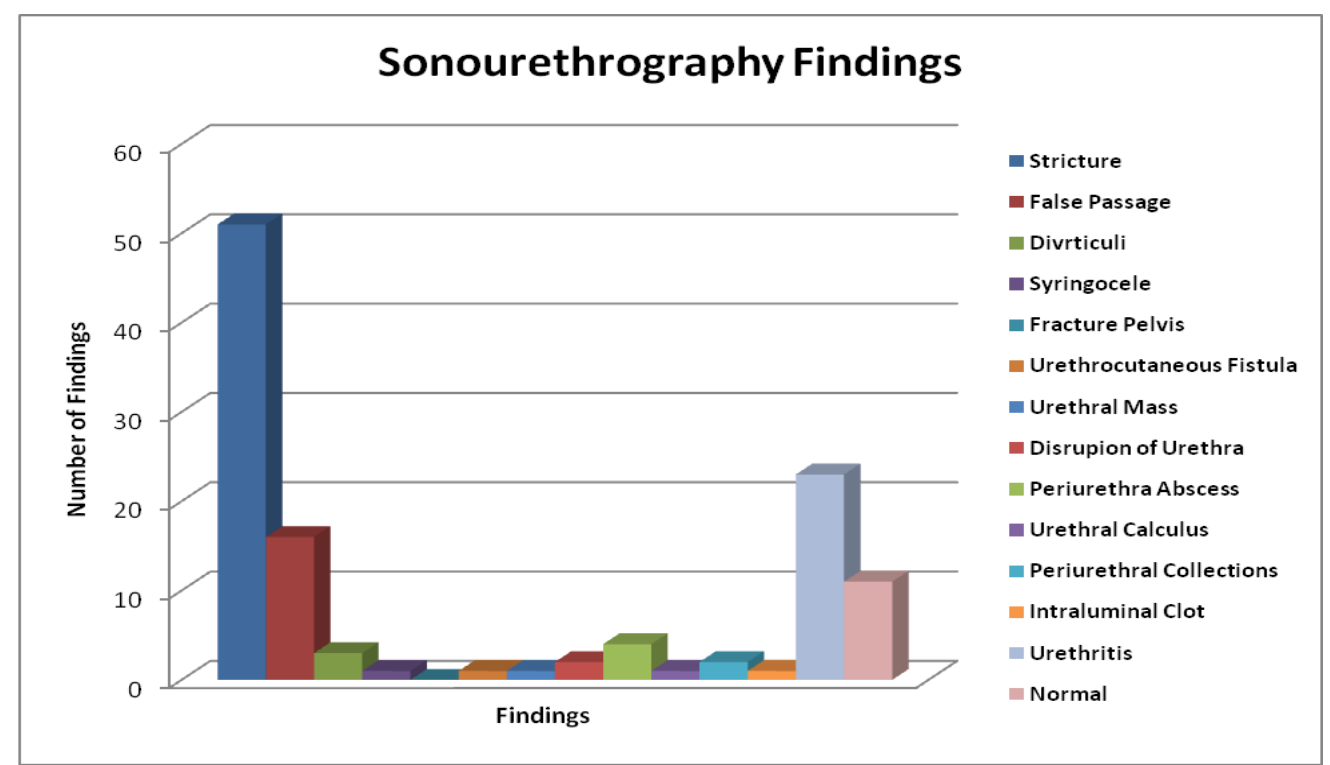

Fig-4: Sonourethrography Findings in the patients with urinary complaints. 


\section{Discussion}

There are considerable numbers of patients with urethral problems and many of them are required to undergo surgical procedures for the treatment of their diseases. Thus preoperative investigation, which would accurately demonstrate and locate the urethral pathology, becomes a prerequisite for successful treatment. If such an investigation is radiation free and able to suggest the mode of treatment, then it virtually becomes indispensable. Sonourethrography is one such investigation.

For this study, the sonographic imaging was performed with the urethra distended by normal saline as negative contrast agent. Bearcroft P.W.P. and Berman L.H. [3] used radiographic contrast medium immediately after the contrast study. If no contrast study was to be performed it was replaced by normal saline.

Previous studies had described dorsal scanning approach to the penile urethra. [2, 4, 5] followed by ventral scanning for the subscrotal and perineal views of the bulbar urethra. These studies however used $5 \mathrm{MHz}$ transducer and near-field artifact was a problem. Despite the penile urethra appearing in the extreme near field, we found that ventral approach was preferable with the penis extended along the lower abdomen. This enabled the longitudinal scans to be accomplished in a single rapid sweep. Near field artifact was abolished by using a high frequency probe $(10 \mathrm{MHz})$.

Out of 60 patients, most belonged to age group 31-40 years. Average age of the patients in our study was 36.33 year. Gluck C.D. et al, Gupta S. et al, Bearcroft P.W.P. et al, Chiou R.K. et al, Samaiyar S.S. et al. $[3,4,6,7,8]$ studied patients for anterior urethral pathologies with sonourethrography and had most patients of the same age group.

\section{IV.i Urethral Strictures}

During SUG, on injection of saline, the urethra distended and appeared as homogenous echo-free band, $8-10 \mathrm{~mm}$ in diameter. Below the urethra an echogenic band was visualized, which was produced by dorsal acoustic enhancement and reflection from tunica albuginea. Strictures were described as segments of reduced distensibility on injection of saline. Total 51 strictures were demonstrated in 44 patients. Out of 44 patients single stricture was found in 31 patients and multiple or complex strictures in 13 patients.

The accurate estimation of stricture length is important, as it is one of the factors that determine the suitable operative procedure. Earlier investigators, using standard radiographic imaging alone, proposed that only strictures $1 \mathrm{~cm}$ or less be selected for excision therapy. [9] Since sonographic measurements are often longer than the actual length, new ultrasonic criteria proposed indicated resection and end-to-end anastomosis for adult bulbar stricture measuring up to $25 \mathrm{~mm}$. We categorized stricture length as short strictures less than 25 $\mathrm{mm}$ in length and long strictures, more than $25 \mathrm{~mm}$ in length. This classification is modification of classification by Chiou R K et al. [7,10] and Morey A. et al. [11].

Out of 44 patients, 28 patients had short segment stricture, 3 patients had long segment stricture and rest 13 patients had complex/multiple strictures. Short segment strictures were seen in 28 patients out of which 22 patients had bulbar short segment stricture and six patients had penile short segment strictures. In long segment strictures total three patients were identified, two with long segment penile stricture (Fig-5) and one with long segment bulbar stricture (Table-2).

Table-2: Distribution of single urethral stricture according to length and location.

\begin{tabular}{|l|c|c|}
\hline Strictures & Short segment & Long segment \\
\hline Penile & 6 & 2 \\
\hline Bulbar & 22 & 1 \\
\hline Total & 28 & 3 \\
\hline
\end{tabular}

Total 13 patients had complex or multiple type of strictures out of which three patients were with multiple penile strictures, seven patients had complex penile and bulbar stricture and three patients had multiple bulbar stricture.

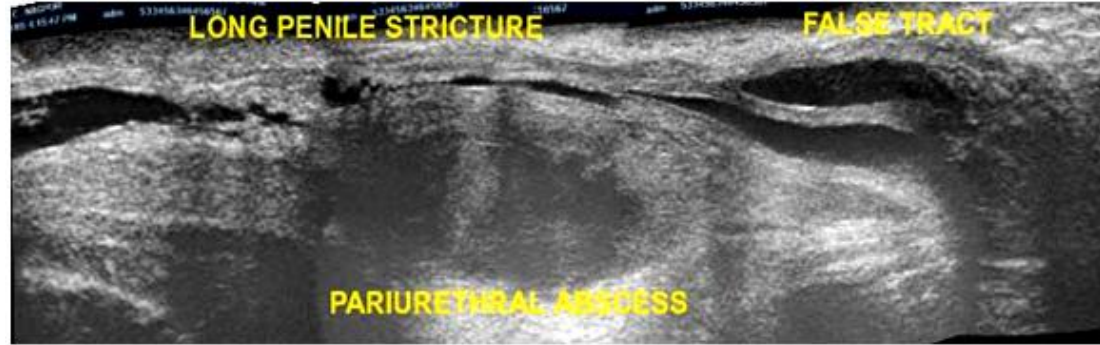




\section{Fig-5: Long penile urethral stricture with periurethral abscess and false tract on sonourethrography.}

\section{IV.ii Periurethral Fibrosis}

Periurethral fibrosis was identified as regions of greater echogenicity in corpus spongiosum, and was classified as per the classification by Chiou R.K. et al $[7,10]$ as.

Minimal spongiosal tissue involvement demonstrates either no identifiable spongy tissue involvement or a minimal abnormality.

Moderate spongiosal tissue involvement shows definite areas of abnormal tissue beneath the urethral surface with sonographically normal tissue in the periphery.

Extensive spongiosal tissue involvement consists of a near full-thickness involvement of the corpus spongiosum.

Out of 44 patients diagnosed with urethral stricture disease, sonography revealed periurethral fibrosis in 34 patients. Mild periurethral fibrosis was seen in seven patients, moderate in 13 patients and extensive in 14 patients. There was no correlation between the presence of PUF and the severity of strictures.

Periurethral fibrosis (spongiofibrosis) is a critical determinant of appropriate therapy and ultimate prognosis. [12]. Excessive fibrosis is said to be responsible for high recurrence rates [5].

\section{IV.iii Other Periurethral Findings}

Other findings such as periurethral haematoma or collection in post trauma patients and periurethral abscesses or masses in patients with infectious etiology were well seen on sonourethrography. Out of 60 patients $6(10 \%)$ patients had periurethral findings other than fibrosis.

Total four patients $(6.66 \%)$ had periurethral abscesses. Two $(3.33 \%)$ patients with trauma had associated periurethral haematoma and collection.

Sonourethrography definitely have upper hand in evaluation of periurethral pathologies. [1]

\section{IV.iv Filling Defects / Intraluminal Findings}

In four patients sonography demonstrated luminal filling defects, one case each of urethral mass, intraluminal blood clot, urethral calculus (Fig-6) and Syringocele with debris within (Fig-7). Bearcroft P.W.P. et al [3] studied 24 patients, 11 were normal. In 5 patients sonography demonstrated luminal filling defects: a mucosal tag, a flap, a urethral papilloma and two cases of debris within a diverticulum. Gluck et al. [4] successfully demonstrated urethral strictures utilizing sonography. Although filling defect was not evaluated in their study, an intraluminal blood clot was well seen in one of their illustrations of straddle injury. Most of the previous studies underestimated these findings.

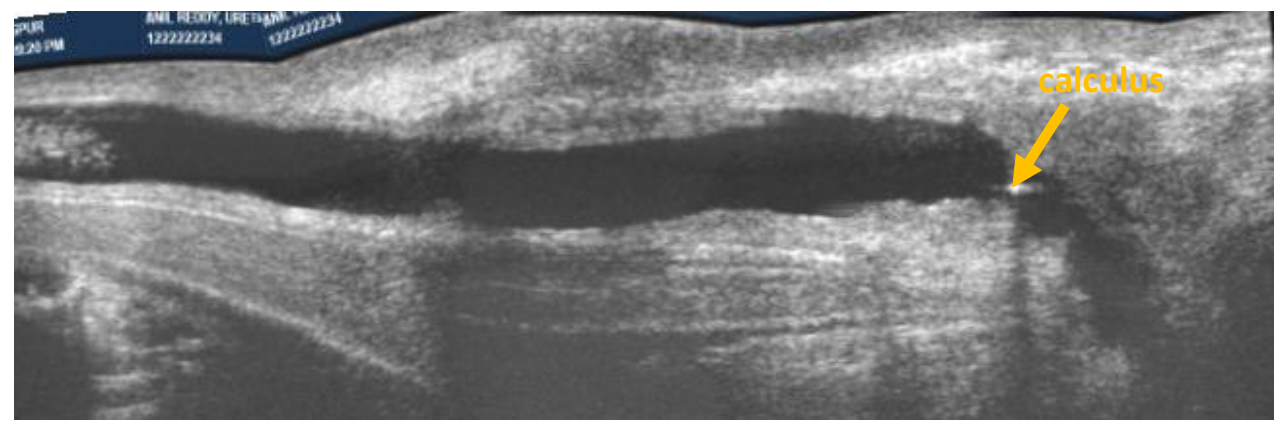

Fig-6: Patient with urethral \& associated short segment bulbar urethral stricture.

\section{IV.v Diverticula}

Total 4 cases of diverticula were diagnosed. Out of these, two wide-mouthed diverticula were posttraumatic. One shallow penile urethral diverticulum was seen in post-traumatic patient with bulbar urethral disruption. A case of syringocele was considered under the heading of diverticula as it has tendency to open in the urethral lumen to give appearance of diverticulum. Syringocele or cystic dilatation of cowper's gland duct is a lesion of uncertain origin even if it is probably congenital since it is found in young patients with negative clinical history. Syringocele can be easily diagnosed by sonourethrography using transperineal scanning which shows a thin septation in the bulbar urethra dividing the distended lumen of the urethra in two parts. The one nearest the probe corresponds to the dilated cowper's duct (Fig-7) [13].

Bearcroft P.W.P. et al [3] studied 24 patients, 11 were normal. Three cases were demonstrated to be of wide mouth diverticula however only two of these were seen on ultrasound. A shallow diverticulum was found in case of multiple strictures. Alanen A, Nurmi M. [14] studied sixteen male patients out of which one patient 
showed diverticulum. Most of the previous studies underestimated these findings.

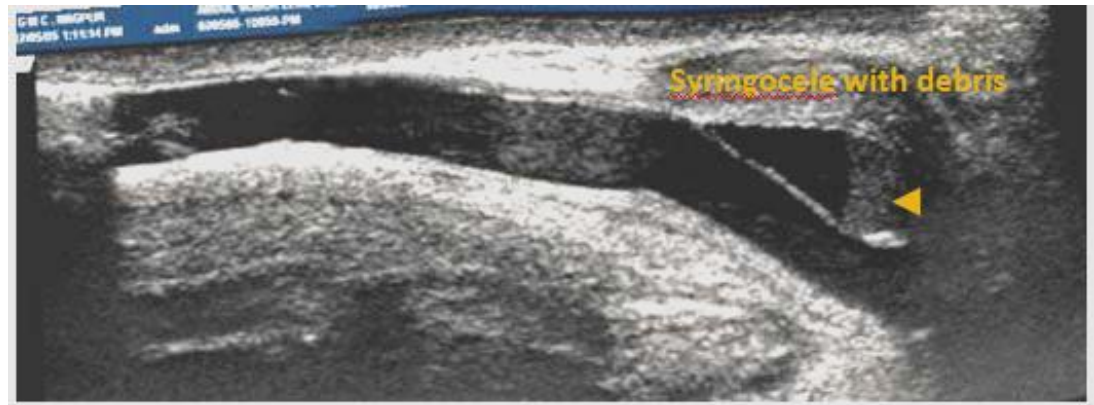

Fig-7: Patient with Syringocele as appearing on Sonourethrography with debris within.

\section{IV.vi Generalised Mucosal Irregularity (Urethritis)}

Generalised mucosal irregularity was observed in 23 patients. Since most of these patients had history suggestive of chronic urethritis, they were presumed to be having urethritis (Fig-8).

Bearcroft P.W.P. et al [3] studied 24 patients, 11 were normal. Total five cases of generalized mucosal irregularity were demonstrated on the sonogram. Most of the previous studies underestimated these findings as these were not much important from management point of view.

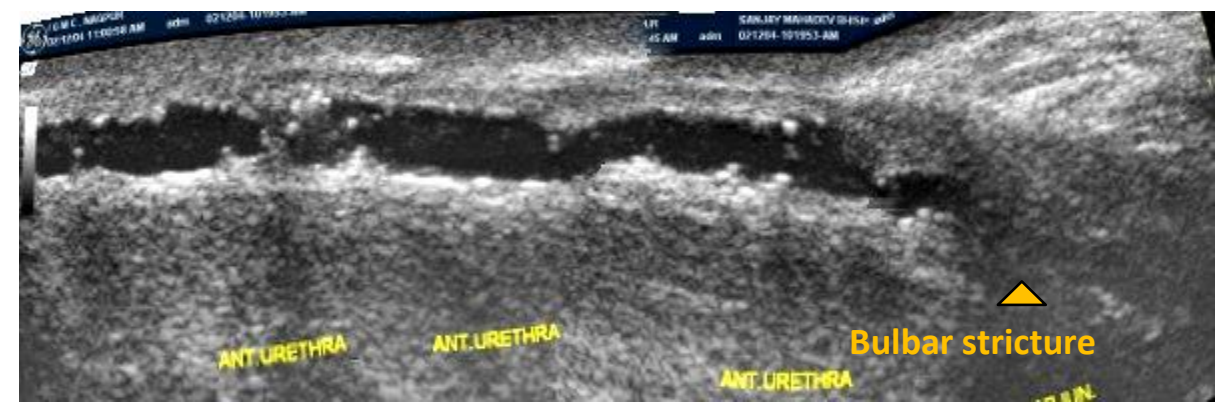

Fig-8: Patient with penile urethral mucosal irregularity and bulbar stricture on Sonourethrography.

\section{IV.vii False Passages}

Careful evaluation of the periurethral structures and proper transverse scanning is needed to demonstrate false passages on sonourethrography. False passages were seen in 16 patients. One patient had urethrocutaneus fistula. Out of 13 patients with strictures, five patients had history of instrumentation and rest of the patients had history of trauma. Two patients had associated periurethral abscesses. Three patients had only false passage without any stricture and had history of trauma.

Chiou RK et al [10] in their study, studied 35 patients, eight patients had inadequate evaluation. Among 27 patients one patient had urethrocutaneus fistula without stricture while another patient had false passage with stricture. Gupta s. et al, [6] were able to pick up 8 false tracts out of 10 on sonourethrography.

During SUG, pain was experienced by one patient during inflation of the Foley's bulb in the fossa navicularis and bleeding per urethra in one patient.

\section{Conclusion}

In the present study we have found sonourethrography to be a simple portable, dynamic, multiplanner, easily available and cost effective technique for evaluating the male anterior urethra without radiation exposure. It is an effective combination of high-resolution sonography and normal saline as a negative contrast agent.

Sonourethrography gives more accurate information about periurethral fibrosis associated with strictures and other abnormalities thus is more useful when determining the type of operative procedure suitable for patients with pathologies especially strictures localized to the anterior urethra

In conclusion, sonourethrography in experienced hand proves to be highly effective modality for diagnosis of anterior urethral pathologies.

\section{References}

[1]. Pavlica P, Barozzi L, Menchi I. Imaging of male urethra. Eur Radiol. 2003 Jul; 13(7): 1583-96.

[2]. McAninch JW, Laing FC, Jeffrey RB Jr. Sonourethrography in the evaluation of urethral strictures: a preliminary report. J Urol. 1988; 139: 294-297. 
[3]. Bearcroft PW, Berman LH. Sonography in the evaluation of the male anterior urethra. Clin Radiol. 1994 Sep; 49(9): 621-6.

[4]. Gluck CD, Bundy AL, Fine C, Kevin RL and Jerome PR. Sonographic urethrogram: comparison to roentgenographic techniques in 22 patients. J Urol. 1988; 140(6): 1404-1408.

[5]. Merkle W, Wagner W. Sonography of the distal male urethra-a new diagnostic procedure for urethral strictures: results of a retrospective study. J Urol. 1988; 140:1409-1411

[6]. Gupta S, Majumdar B, Tiwari A, Gupta RK, Kumar A, Gujral RB. Sonourethrography in the evaluation of anterior urethral strictures: correlation with radiographic urethrography. J Clin Ultrasound. 1993; 21(4): 231-239.

[7]. Chiou RK, Donovan JM, Anderson JC, Matamoros A Jr; Wobig RK, Taylor RJ. Color Doppler ultrasound assessment of urethral artery location: potential implication on visual internal urethrotomy. J Urol. 1998; 159:796-9.

[8]. Samaiyar SS; Shukla RC; Dwivedi US. Role of sonourethrography in anterior urethral stricture. Indian Journal of Urology. 1999 Mar; 15(2): 146-51.

[9]. Webster G.D., Koefoot R.B. and Sihelnik S.A. Urethroplasty management in 100 cases of urethral stricture: a rationale for procedure selection. J Urol 1985; 134 p. 892.

[10]. Chiou RK, Anderson JC, Tran T, Ptterson RH, Wobig R, and Taylor RJ. Evaluation of urethral strictures and associated abnormalities using high-resolution and color Doppler ultrasound. Urology. 1996; 47:102-107.

[11]. Morey AF, McAninch JW. Ultrasound evaluation of the male urethra for assessment of urethral stricture. J Clin Ultrasound 1996; 24:473-9.

[12]. Jordan G.H., Schlossberg S.M. and Devine C.J. Surgery of the penis and urethra. In: Walsh F.C., Retik A.B., Vaughan E.D. Jr et al.Campbell's urology (7th ed.), WB Saunders, Philadelphia .1998; pp. 3318-3394.

[13]. Merchant SA, Amonkar PP, Patil JA. Imperforate syringoceles of the bulbourethral duct: appearance on urethrography, sonography and CT. Am J Roentgenol 1997; 169:823-824.

[14]. Alanen A, Nurmi M. Sonographic technique in diagnosis of urethral strictures in men. Bildgebung. 1994 Mar; 61(1): 25-7. 